\title{
Histologic analysis and lipid profiling reveal reproductive age-associated changes in peri-ovarian adipose tissue
}

\author{
Shweta S. Dipali', Christina R. Ferreira ${ }^{2,3}$, Luhan T. Zhou ${ }^{1}$, Michele T. Pritchard ${ }^{4}$ and Francesca E. Duncan ${ }^{1 *}$ (D)
}

\begin{abstract}
Background: Reproductive aging is a robust phenotype that occurs in all females and is characterized by a significant reduction in gamete quantity and quality, which can have negative consequences on both endocrine function and fertility. Age-associated differences in the oocyte, follicle, and ovary have been well-documented, but how the broader environment changes with age is less well understood. Fat is one of the largest organs in the body, and peri-gonadal adipose tissue surrounds the rodent ovary and comprises a local ovarian environment. The goal of this study was to characterize how peri-ovarian adipose tissue changes with advanced reproductive age.

Methods: We isolated peri-gonadal adipose tissue from two cohorts of CB6F1 mice: reproductively young (6-12 weeks) and reproductively old (14-17 months). A comparative histological analysis was performed to evaluate adipocyte architecture. We then extracted lipids from the tissue and performed multiple reaction monitoring (MRM)profiling, a mass spectrometry-based method of metabolite profiling, to compare the lipid profiles of peri-gonadal adipose tissue in these age cohorts.
\end{abstract}

Results: We found that advanced reproductive age was associated with adipocyte hypertrophy and a corresponding decrease in the number of adipocytes per area. Of the 10 lipid classes examined, triacylglycerols (TAGs) had significantly different profiles between young and old cohorts, despite quantitative analysis revealing a decrease in the total amount of TAGs per weight of peri-gonadal adipose tissue with age.

Conclusions: These findings pinpoint age-associated physiological changes in peri-gonadal adipose tissue with respect to adipocyte morphology and lipid profiles and lay the foundation for future studies to examine how these alterations may influence both adipocyte and ovarian function.

Keywords: Adipose, Peri-gonadal, Triacylglycerol, Ovary, Aging

\section{Background}

Aging is characterized by progressive physiological deterioration at both the cellular and tissue levels. This deterioration causes impaired function and is one of the primary risk factors for many human diseases [1]. The female reproductive system is unique in that it is the first organ system in the body to age. Female reproductive aging is marked by decreased egg quality and quantity that results in decreased fertility and a loss of endocrine function [2]. Women of advanced reproductive age have a

\footnotetext{
* Correspondence: f-duncan@northwestern.edu

${ }^{1}$ Department of Obstetrics and Gynecology, Feinberg School of Medicine, Northwestern University, 303 E. Superior Street, Lurie 7-117, Chicago, IL 60611, USA

Full list of author information is available at the end of the article
}

higher risk of miscarriage and/or of having offspring with genetic defects, which is a growing problem as more women worldwide are delaying child-bearing $[3,4]$.

The age-associated decline in gamete quality is multi-factorial and has been attributed to changes at the level of the gamete, the follicle, and the immediate stromal environment. Reproductive aging in the oocyte is correlated with alterations in chromosomes, mitochondria, nucleoli, ribosomes, microtubules, spindle poles, and transzonal projections [5-11]. The oocyte does not develop in isolation but is instead highly dependent on surrounding granulosa cells within the intact ovarian follicle. Follicles isolated from reproductively old mice exhibit altered gene expression patterns and hormone

(C) The Author(s). 2019 Open Access This article is distributed under the terms of the Creative Commons Attribution 4.0 International License (http://creativecommons.org/licenses/by/4.0/), which permits unrestricted use, distribution, and 
profiles and contain oocytes with reduced meiotic competence, including a higher incidence of spindle defects, relative to reproductively young controls $[6,12]$. Moreover, the growth of ovarian follicles is dependent on the stromal microenvironment which includes a highly structured extracellular matrix (ECM), fibroblasts, smooth muscle cells, endothelial cells, theca-interstitial cells, and immune cells. This ovarian microenvironment changes with advanced reproductive age and is associated with increased fibrosis, the presence of a unique population of multinucleated macrophage giant cells, and elevated expression of inflammatory cytokines [13]. How the physiology of the environment immediately surrounding the ovary changes with age has not been carefully examined.

In rodents, the ovary is surrounded by visceral fat pads in the peri-gonadal region $[14,15]$. This periovarian fat depot does not exist in humans but is most highly analogous in function to the visceral omental fat [14]. Adipose tissue plays an important role in energy storage, temperature regulation, and mechanical protection, in addition to having several immune and endocrine functions [14]. The majority of cells in adipose tissue are adipocytes, which store energy as fat within lipid droplets, but fat tissue also contains pre-adipocyte progenitor cells, fibroblasts, endothelial cells, pericytes, and immune cells [16]. Aging is associated with a shift of adipose tissue from the periphery to a more central deposition, especially in the viscera, which contributes to increased metabolic disease in adults [17-19]. With age, fat tissue metabolism defects occur ranging from preadipocyte dysfunction to the inability of fat cells to properly store or release energy or respond to excess lipotoxic fatty acids [20]. Furthermore, aging is also associated with cellular senescence and chronic, low-grade inflammation in adipose tissue with increased local and circulating proinflammatory cytokines $[20,21]$.

The goal of our study was to examine whether changes in periovarian adipose tissue architecture and lipid profiles are detectable at a developmental window known to be associated with established reproductive aging phenotypes, including decreased ovarian reserve, increased egg aneuploidy, and increased ovarian stromal fibrosis [6, 8, 13, 22-25]. We expect that age-associated changes in adipose tissue may impact ovarian function given the established connections between lipids, the ovary, and the oocyte. For example, the composition and metabolism of lipids within ovarian follicles, both in follicular fluid and as lipid droplets within the oocytes, impacts the developmental competence of the oocyte [26, 27]. Moreover, the lipid profile in human follicular fluid changes with age [28]. Altered lipid profiles within the ovary are also characteristic of disease states. Ovaries from rabbits with hypothyroidism have increased total cholesterol and glycogen content, but reduced triacylglycerol content in comparison to control rabbits [29]. Moreover, hypothyroidism reduces the size of follicles in the rabbit ovary, in addition to inducing hypertrophy of adipocytes and infiltration of macrophages into periovarian adipose tissue, together suggesting interplay between the adipose tissue and the ovary [30].

Through a comparative histological analysis of peri-gonadal adipose tissue of reproductively young and old mice, we found that advanced reproductive age was associated with adipocyte hypertrophy and a corresponding decrease in the number of adipocytes per area. We then used multiple reaction monitoring (MRM)-profiling, a mass spectrometry based method of metabolite profiling that allows accelerated discovery of a large number of discriminating molecular features, to compare the lipid profiles of peri-gonadal adipose tissue from reproductively young and old mice [31, 32]. Of the 10 lipid classes examined, triacylglycerols (TAGs) had significantly different profiles between young and old cohorts. Interestingly, however, quantitative analysis revealed that there was a decrease in the total amount of TAGs per weight of peri-gonadal adipose tissue with age. Overall, this study pinpoints key physiological differences that occur with age in peri-gonadal adipose tissue and sets the stage for future studies to explore the consequences of such changes on reproductive aging, especially given the close proximity of the adipose tissue to the ovary and oocytes.

\section{Methods}

Animals and peri-gonadal adipose tissue collection

Reproductively young CB6F1 mice (6-12 weeks old) were obtained from Envigo (Indianapolis, IN) and reproductively old mice (14-17 months old) were obtained from the National Institute on Aging Aged Rodent Colony (National Institutes of Health, Bethesda, MD). Based on a linear extrapolation of age, the 14-17 month old cohort corresponds to women in their late thirties to early forties. Studies in several strains across multiple laboratories have demonstrated that female mice at this age exhibit reproductive aging phenotypes $[6,8,10,13,22-25,33]$. Studies with CB6F1 mice within the same age cohorts demonstrate a significant age-associated decrease in the number of primordial follicles or ovarian reserve as well as an increase in egg aneuploidy $[6,8]$. All mice were housed in a controlled barrier facility at Northwestern University's Center for Comparative Medicine on the Chicago Campus under constant temperature, humidity, and light (14 h light/10 h dark). The mice in the different age cohorts were fed specific chows in their respective source facilities, but upon arrival to Northwestern University, water and Teklad Global irradiated 2016 chow containing minimal phytoestrogens and no soybean or alfalfa meal (Envigo, Madison, WI) were provided ad libitum (Additional file 1: Table S1). A summary of the fat content and fatty acid composition of 
the chows is listed in Additional file 1: Table S1. All animal experiments described here were approved by the Institutional Animal Care and Use Committee (Northwestern University) and were performed in accordance with National Institutes of Health Guidelines.

Due to the limited availability of reproductively old animals, we analyzed peri-gonadal adipose tissues from a cohort of reproductively young and old animals that were both part of a separate study involving superovulation. Both reproductively young and old mice received an intraperitoneal (IP) injection of 5 IU pregnant mare serum gonadotropin (PMSG, EMG Millipore, Burlington, MA) followed $44-46 \mathrm{~h}$ by an IP injection of $5 \mathrm{IU}$ human chorionic gonadotropin (hCG, Sigma-Aldrich, St. Louis, MO), and tissue was collected $14-16 \mathrm{~h}$ post-hCG injection. Serum progesterone levels were not significantly different in reproductively young and old CB6F1 mice following hyperstimulation (data not shown). Mice were weighed prior to euthanasia, and adipose tissue from the peri-ovarian fat pad that surrounds the ovary was carefully dissected from both reproductively young and old mice (Fig. 1a). The isolated samples were either fixed for histochemical analysis or snap frozen for mass spectrometry (MS) and MRM-profiling as described in more detail below.

\section{Histochemical analysis}

Tissue samples were washed in $1 \mathrm{X}$ phosphate buffered saline and fixed in Modified Davidson's (Electron Microscopy Sciences, Hatfield, PA) for $16 \mathrm{~h}$ at $4{ }^{\circ} \mathrm{C}$. Samples were transferred to $70 \%$ ethanol until processing, dehydrated using an automated tissue processor (Leica Microsystems), and embedded in paraffin. Sections were cut at a $5 \mu \mathrm{m}$ thickness. Tissue sections were deparaffinized in Citrisolv (Fisher Scientific, Pittsburgh, PA) and then rehydrated in a series of graded ethanol baths $(100$, 70 , and $30 \%$ ). The deparaffinization process washes away lipids leaving behind the extracellular matrix which can be used to visualize the border of the adipocytes with a histological stain such as Picrosirus Red (PSR) which stains collagen I and III. To perform the PSR staining, slides were immersed in a staining solution prepared by dissolving Sirius Red F3B (Direct Red 80, C.I. 35,780, Sigma-Aldrich, St. Louis, MO) in a saturated aqueous solution of picric acid (Sigma-Aldrich, St. Louis, MO) at $0.1 \% \mathrm{w} / \mathrm{v}$. Slides were incubated in the PSR staining
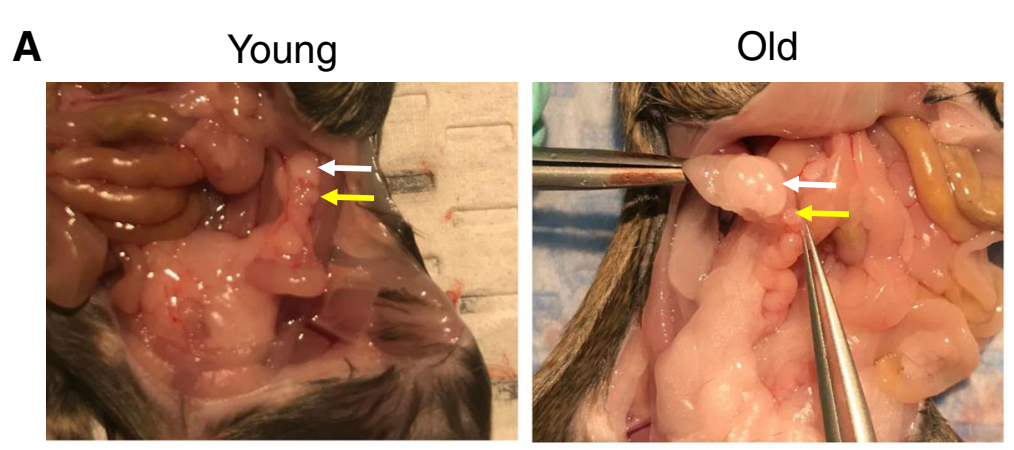

B
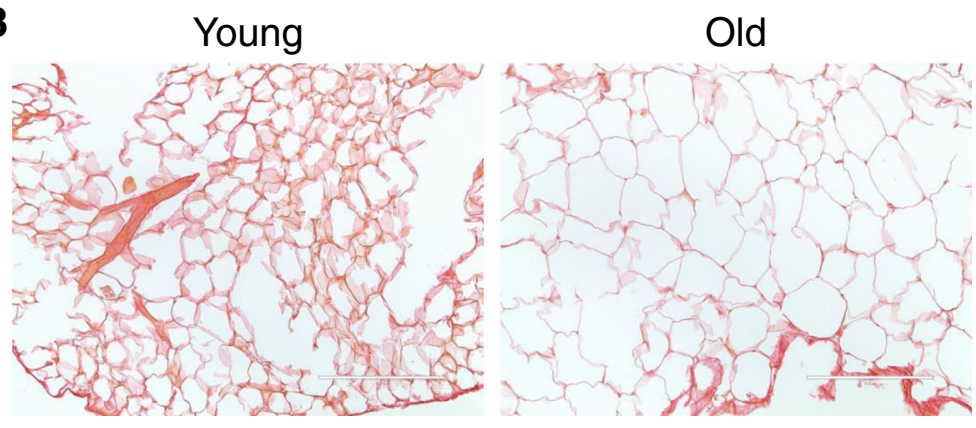

\section{C} Average Adipocyte Cross-Sectional Area

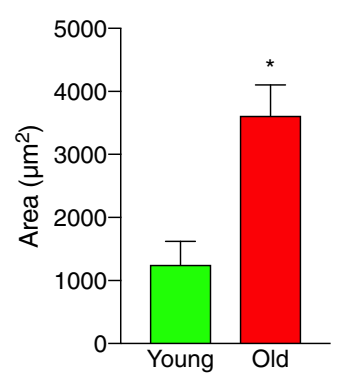

D

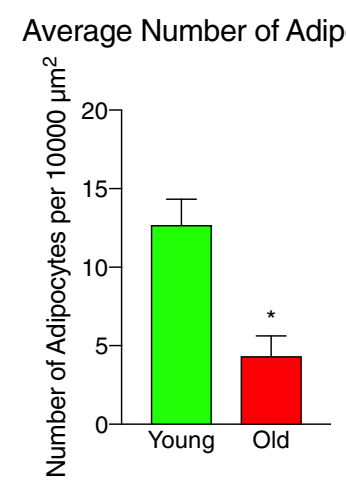

Fig. 1 Adipocyte size in peri-gonadal adipose tissue increases with advanced reproductive age. a Representative anatomical images showing the ovary (yellow arrow) and the surrounding peri-gonadal adipose tissue (white arrow) in reproductively young and old mice. $\mathbf{b}$ Representative images of histological sections of peri-gonadal adipose tissue from reproductively young and old mice stained with PSR. The scale bars $=200 \mu \mathrm{m}$. c The average cross-sectional adipocyte area $(\mu \mathrm{m} 2)$ in peri-gonadal adipose tissue from reproductively young (green) and old (red) mice. b The average number of adipocytes per $10,000 \mu \mathrm{m} 2$ in peri-gonadal adipose tissue from reproductively young (green) and old (red) mice. Error bars represent standard error between samples. ${ }^{*} P$ value $\leq 0.05$ by t-test. 
solution for $40 \mathrm{~min}$ at room temperature followed by a wash in $0.05 \mathrm{M}$ hydrochloric acid (Fisher Scientific) for 90 s. Excess acidified water was carefully wicked away from the tissue sections, and the tissue was rapidly dehydrated in 100\% ethanol (3, $30 \mathrm{~s}$ incubations). The slides were cleared in fresh Citrisolv for five minutes and mounted using Cytoseal XYL (Fisher Scientific). All slides used for PSR staining were processed at the same time to minimize variation in staining between sequential assays.

\section{Adipose tissue imaging and analysis}

Brightfield images were taken with an EVOS FL Auto Cell Imaging system (Thermo Fisher, Waltham, MA) using $20 \mathrm{X}$ or $40 \mathrm{X}$ objectives. To view entire adipose tissue sections, scans comprised of a series of individual images were taken across the tissue and then automatically stitched together using the EVOS software. Light settings were kept consistent between images of different tissue sections, and post-processing adjustment of brightness and contrast was done equivalently across samples to improve the clarity of the images and did not impact data analysis. Adipocytes were clearly visible due to their outline by PSR-positive staining. To quantify adipocyte number, three random areas per adipose tissue section were defined using the EVOS software, and the number of adipocytes per area was counted. We also measured the cross-sectional area of 50 adipocytes per adipose tissue section using the EVOS software, with the borders of each adipocyte defined by the PSR staining. This analysis was performed on peri-gonadal adipose tissue samples from 2 reproductively young and 3 reproductively old mice.

\section{Lipid extraction}

For MS analysis, peri-gonadal tissue samples from 5 mice per age group were sent to the Metabolite Profiling Facility at Purdue University for MRM-profiling. The tissue from both the right and left ovaries was combined for each animal and weighed. Lipids were extracted using the Bligh \& Dyer extraction method [34]. In brief, samples were transferred to a $2 \mathrm{~mL}$ vial with inert 1.4 $\mathrm{mm}$ ceramic (zirconium oxide) beads (Precellys CK 14, Bertin Corp, part \# P000912-LYSK0A), and $500 \mu \mathrm{L}$ of ultrapure water was added to homogenize the sample using the Precellys tissue homogenizer (Bertin Corp, Rockville, MD, US) at three cycles of $6200 \mathrm{rpm}$ for $30 \mathrm{~s}$. Next, $300 \mu \mathrm{L}$ of homogenized tissue was transferred to a new microtube and mixed with $250 \mu \mathrm{L}$ of chloroform and $450 \mu \mathrm{L}$ of methanol. This solution was incubated at room temperature for $15 \mathrm{~min}$. After that, $250 \mu \mathrm{L}$ of chloroform and $250 \mu \mathrm{L}$ of water were added and the sample was centrifuged for $10 \mathrm{~min}$ at $16,000 \mathrm{x} \mathrm{g}$, forming a 2-phase solution where the bottom phase contained the lipids (organic phase). The organic phase was transferred to a new tube and dried using a speedvac centrifuge (Savant Speedvac, Thermo Scientific Inc., San Jose, CA, US), and samples were stored at $-80^{\circ} \mathrm{C}$ until mass spectrometry analysis.

\section{Targeted lipid profiling}

Targeted lipid profiling was performed using discovery MRM-profiling methods and instrumentation as recently described [32]. Specifically, for sample preparation, dried lipid extracts were diluted in $50 \mu \mathrm{L}$ of methanol/chloroform 3:1 (v/v) and $250 \mu \mathrm{L}$ of injection solvent (acetonitrile/methanol/ammonium acetate $300 \mathrm{mM}$ 3:6.65:0.35 $(\mathrm{v} / \mathrm{v}))$ to obtain a stock solution. Mass spectrometry data was acquired by flow-injection (no chromatographic separation) from $8 \mu \mathrm{L}$ of diluted lipid extract stock solution delivered using a micro-autosampler (G1377A) to the ESI source of an Agilent 6410 triple quadrupole mass spectrometer (Agilent Technologies, Santa Clara, CA, USA). A capillary pump was connected to the autosampler and operated at a flow rate of $20 \mu \mathrm{L} / \mathrm{min}$ and pressure of 150 bar. Capillary voltage on the instrument was $3.5-5 \mathrm{kV}$ and the gas flow $5.1 \mathrm{~L} / \mathrm{min}$ at $300^{\circ} \mathrm{C}$. For the data acquisition, further dilution of the lipid extract stocks according to the sample weight was performed so that each injection corresponded to approximately $1 \mu \mathrm{g}$ of tissue.

The comparative analysis of the amount of phosphatidylcholine (PC), sphingomyelin (SM) and triacylglycerol (TAG) lipids between old and young animals was estimated after spiking one representative sample (containing a mixture of the lipid extracts of the 5 animals) for young and one for old animals with SPLASH ${ }^{\circ}$ LIPIDOMIX $^{\circ}$ Mass Spec Standard (Avanti Polar Lipids Inc., Alabaster, AL, US) and running the lipid profile method for these lipid classes including the MRM pair for 15:018:1(d7) PC, 18:1(d9) SM, and 15:0-18:1(d7)-15:0 TG for estimating the amount of PC, SM and TAG, respectively, in the two samples. Three analytical replicates were used for the data analysis.

\section{Statistical analysis}

For the histochemical analysis, the average adipocyte area and average number of adipocytes per $10,000 \mu \mathrm{m}^{2}$ were graphed using Graphpad Prism Software Version 8.0.1 (La Jolla, CA). Significant changes between groups were analyzed by students' t-test and $P$ values $<0.05$ were considered statistically significant. For the MS analysis, relative amounts of ion abundances were used for statistics. Values of ion intensities for each of the MRMs monitored were normalized by total ion intensity of all MRMs in the method for a given sample. Further statistical analysis was then performed using Metaboanalyst 3.0 software (https://www.metaboanalyst.ca). Uploaded 
data was auto-scaled and volcano plots (Fold change threshold 1.5, $P$ value threshold 0.05 ), principal component analyses (PCAs), and heatmaps (for top $25 \mathrm{MRMs}$ ) were plotted. Fold change was calculated by dividing values of ion intensities for each of the MRMs measured in each sample by the ion intensity of the corresponding MRM in the blank. Average fold change was graphed for significantly different lipid species using Graphpad Prism Software Version 8.0.1. Significant changes between groups were analyzed by students' t-test and $P$ values < 0.05 were considered statistically significant.

\section{Results}

\section{Adipocyte size increases within peri-gonadal adipose tissue with advanced reproductive age}

Advanced reproductive age was associated with increased animal weight. Reproductively old mice weighed an average of $34.8 \pm 1.89 \mathrm{~g}$ compared to reproductively young mice which weighed $21.7 \pm 0.62 \mathrm{~g}(P=0.0002)$. This difference in total animal weight also correlated with an increase in the amount of peri-gonadal fat that surrounded the ovary that was visible by eye and also reflected in the weight of the tissue collected (Fig. 1a). We collected $195.7 \pm 40.60 \mathrm{mg}$ of peri-gonadal adipose tissue from reproductively old mice compared to $65.3 \pm$ $11.79 \mathrm{mg}$ in reproductively young mice $(P=0.015)$. Within the peri-gonadal adipose tissue, we noted a prominent increase in adipocyte size that occurred with advanced reproductive age (Fig. 1b-c). The average cross-sectional area of adipocytes in peri-gonadal fat from reproductively old mice was $3613.64 \pm 490.51 \mu \mathrm{m}^{2}$ compared to $1254.03 \pm 368.94 \mu^{2}$ in reproductively young mice (Fig. 1c). Consistent with this, the average number of adipocytes per area was significantly lower in peri-gonadal adipose tissue from reproductively old mice as compared to reproductively young mice $(4.34 \pm 1.28$ adipocytes/ $10,000 \mu \mathrm{m}^{2}$ vs $12.69 \pm 1.64$ adipocytes $/ 10,000 \mu \mathrm{m}^{2}$, respectively; Fig. 1d). These results suggest that advanced reproductive age is associated with adipocyte hypertrophy.

\section{Acyl-carnitine, phosphatidylcholine and sphingomyelin, and phosphatidylinositol lipid profiles in peri-gonadal adipose tissue cluster based on age}

To determine if there were differences in lipid profiles that occur with age, we extracted lipids from peri-gonadal adipose tissue from reproductively young and old mice and performed MRM-profiling. This method enabled the interrogation of the relative amounts of numerous lipid species within ten major classes of lipids based on the LipidMaps database. These lipid classes included: triacylglycerols (TAGs), acyl-carnitines, phosphatidylcholines (PCs) and sphingomyelins (SMs), phosphatidylinositols, ceramides, cholesteryl esters, free fatty acids, phosphatidylethanolamines, phosphatidylglycerols, and phosphatidylserines.
Cholesteryl ester, free fatty acid, and phosphatidylglycerol lipid profiles did not have relative ion transition measurements above background, thus preventing any further conclusions about the differences in the profiles between peri-gonadal adipose tissue from reproductively young and reproductively old mice (data not shown). No differences in ceramide, phosphatidylethanolamine, and phosphatidylserine lipid profiles were observed in peri-gonadal adipose tissue with age, and this is evident in the PCA plots for these three classes which showed minimal to no discrimination between samples from reproductively young and old mice (Additional file 2: Figure S1A-C).

Although the PCA plots for acyl-carnitine, phosphatidylcholine and sphingomyelin, as well as phosphatidylinositol lipid profiles did not show significant separation between reproductively young and reproductively old mice (Fig. 2a-c), heatmaps of the 25 most abundant lipid species in each class did show clustering of samples from reproductively young and old mice (Fig. 2d-f, Table 1). This result indicated that certain lipid species in these classes are consistently present in peri-gonadal adipose tissue from reproductively young mice as compared to tissue from reproductively old mice and vice versa.

\section{Triacylglycerol lipid species exhibit significantly different profiles between peri-gonadal adipose tissue from reproductively young and old mice}

Due to the large number of triacylglycerol (TAG) lipid species interrogated in this study, TAGs were run in two separate methods (TAG 1 and TAG 2), each measuring relative ion transitions for different TAG species. The TAG species measured in each method were arbitrarily divided. This analysis revealed that 160 TAG species had significantly different profiles in peri-gonadal adipose tissue from reproductively young and reproductively old mice with a minimum fold-change of 1.5 times between the two ages, and these are highlighted in the volcano plots (Fig. 3a-b, Table 2, Additional file 1: Table S2). Seven of the 160 significantly different TAGs were more abundant in peri-gonadal adipose tissue from reproductively young mice and the remaining 153 were more abundant in tissue from reproductively old mice (Table 2, Additional file 1: Table S2). PCA plots generated based on the significantly different TAGs showed an almost completely distinct separation of the samples by age (Fig. 3c-d). Heatmaps of the 25 most abundant TAG species measured in each of the two methods showed clustering between samples from reproductively young and old mice (Fig. 3e-f, Table 1). Taken together, these results demonstrate that overall TAG lipid profiles in peri-gonadal adipose tissue differ in an age-dependent manner. 


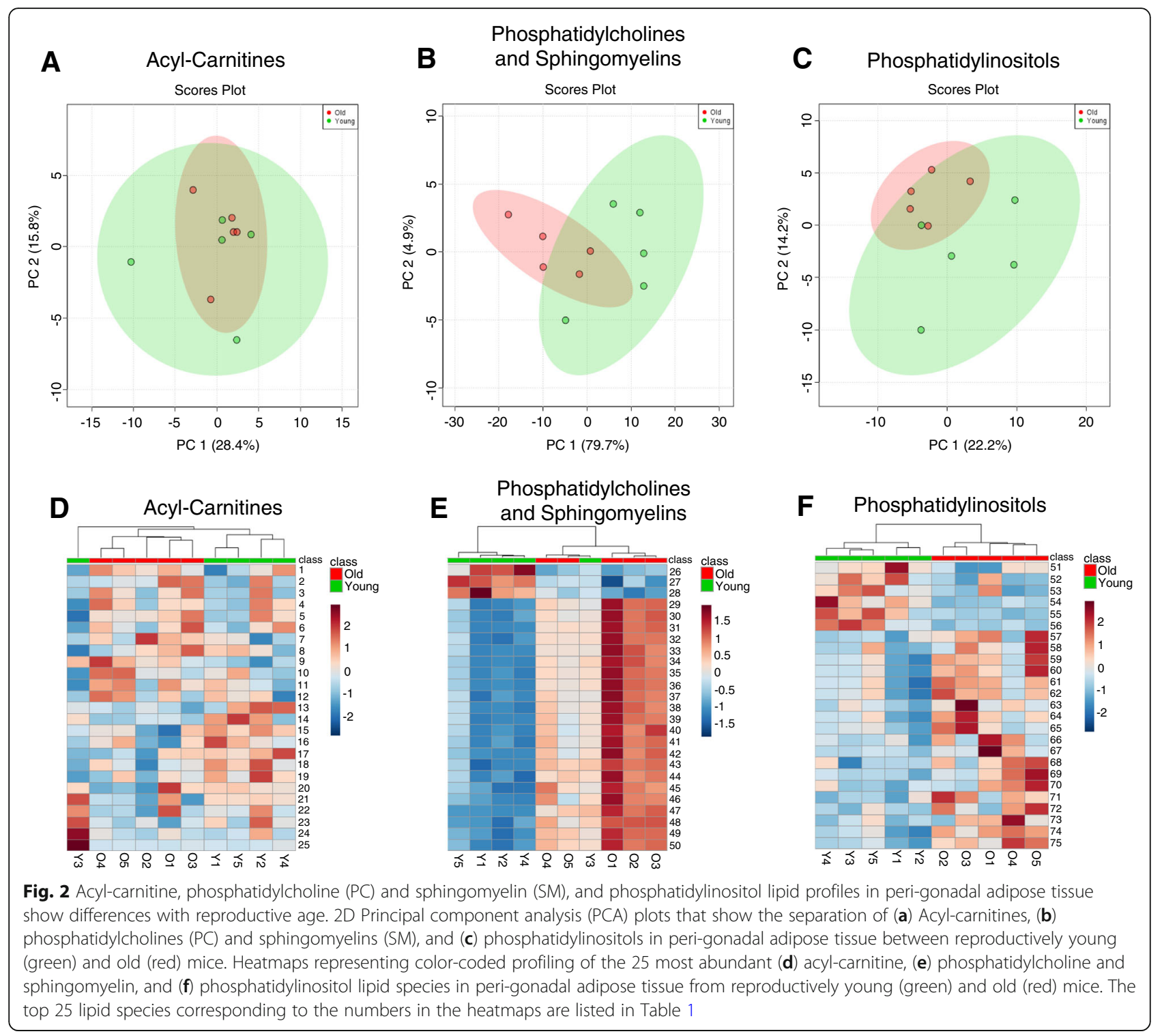

To further characterize the 160 TAGs that were significantly different between reproductively young and reproductively old mice, we analyzed them based on length, unsaturation, and fatty acid composition. When comparing TAGs based on the number of carbons, there were several where the average fold change relative to ion transition measurements taken from a blank sample were decreased in peri-gonadal adipose tissue from reproductively old mice compared to reproductively young mice (Fig. 4a-b). In the TAG 1 method, there was a significant decrease in average fold change in TAGs with 52 and 54 carbons, and in the TAG 2 method, there was a significant decrease in TAGs with 50, 54, and 60 carbons (Fig. 4a-b). In addition to changes in the length of the fatty acid chains, there were also changes in saturation with age (Fig. 4c-d). In the TAG 1 method, the average fold change was significantly less for TAGs that had 4,5 , or 6 sites of unsaturation in peri-gonadal adipose tissue from reproductively old mice compared to young mice (Fig. 4c). The TAG 2 method showed a significant decrease in average fold-change for TAGs with $1,3,4,6$, and 7 sites of unsaturation with advanced reproductive age (Fig. 4e). In the TAG 1 method, there was a significant decrease in average fold-change in TAGs containing palmitic acid (16:0) and stearic acid (18:0) in peri-gonadal adipose tissue from reproductively old mice compared to young mice. (Fig. 4e). In the TAG 2 method, a similar decrease with age was observed in the average fold change for TAGs containing linoleic acid (18:2) and arachidic acid (20:0) (Fig. 4f). These results further underscore the differences in lipid profiles that occur with age in peri-gonadal adipose tissue. 


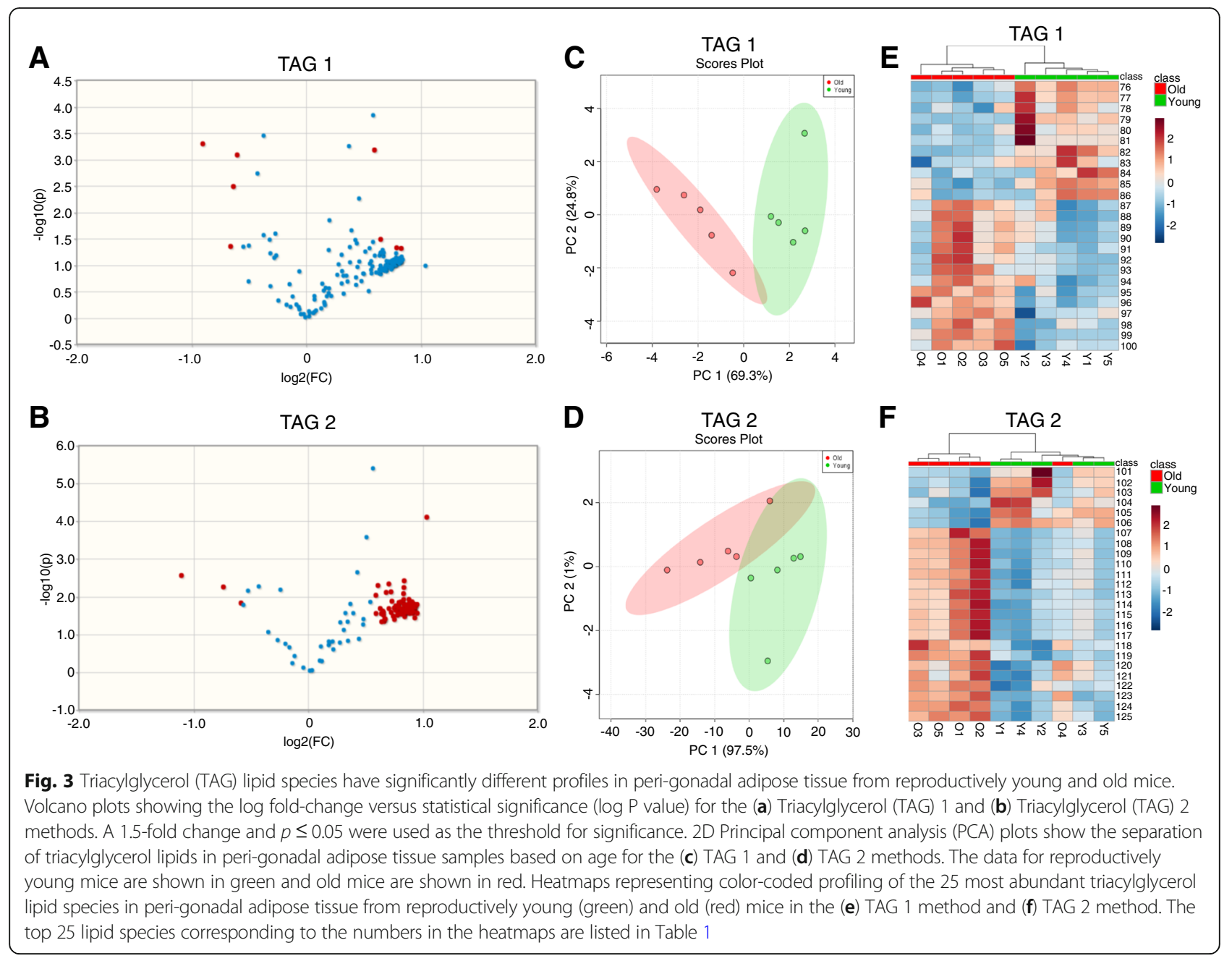

The amount of TAGs per tissue mass quantitatively decreases with age in peri-gonadal adipose tissue

To examine whether the changes in lipid profiles that occur with age are accompanied by quantitative differences in the amounts of the lipids, we compared relative ion transition measurements collected from MRM-profiling of peri-gonadal adipose tissue from reproductively young and old mice to those collected from standards of known concentrations for a given lipid class. We performed this analysis for the TAGs as well as PCs and SMs (Table 3). The PCs and SMs were present in similar quantities in peri-gonadal adipose tissue from reproductively young and old mice, and this conservation across age may be expected given the structural importance of this lipid class to cell membranes. However, the quantity of TAGs decreased with advanced reproductive age from $33.1 \mu \mathrm{g}$ per $\mathrm{mg}$ of adipose tissue in reproductively young mice to $10.2 \mu \mathrm{g}$ per mg of adipose tissue in reproductively old mice (Table 3 ). Consistent with this result, summed relative intensities of TAGs containing linoleic (18:2), palmitic (16:0), palmitoleic (16:1), stearic (18:0), and oleic (18:1) fatty acid residues also decreased with advanced reproductive age (Fig. 5).

\section{Discussion}

In this study, we expanded our knowledge of female reproductive aging by examining how the composition of the adipose tissue surrounding the ovary changes with age. This understanding is significant because fat is one of the largest organs in the body, and this specific depot comprises the local environment of the ovary in the mouse and may, therefore, influence ovarian function on the basis of proximity. Consistent with previous studies, one of the most striking observations we made was the increase in the size of the adipocytes in peri-gonadal adipose tissue with advanced reproductive age. A similar enlargement of adipocytes was observed in epididymal white adipose tissue (WAT) in middle age Wistar rats and also in epididymal and subcutaneous WAT of aged mice $[35,36]$. This adipocyte hypertrophy could be a result of the limited plasticity of adipose tissue that 
Table 1 The 25 most abundant lipid species in each class found in heatmaps (Figs. 2 and 3) ${ }^{a}$

\begin{tabular}{|c|c|}
\hline Number on Heatmap & Lipid Name \\
\hline \multicolumn{2}{|l|}{ Acyl-Carnitines } \\
\hline 1 & 3-hydroxylinoleoylcarnitine \\
\hline 2 & O-behenoylcarnitine \\
\hline 3 & (5Z,8Z)-3-hydroxytetradecadienoylcarnitine \\
\hline 4 & O-adipoylcarnitine \\
\hline 5 & (2E)-hexenedioylcarnitine, O-octanoylcarnitine \\
\hline 6 & O-malonylcarnitine, Hydroxybutyrylcarnitine \\
\hline 7 & Acetyl-carnitine \\
\hline 8 & Butenylcarnitine \\
\hline 9 & Butyrylcarnitine, Isobutyryl-L-carnitine \\
\hline 10 & 3-hydroxyarachidonoylcarnitine \\
\hline 11 & Propenoylcarnitine \\
\hline 12 & (9Z,12Z)-3-hydroxyhexadecadienoylcarnitine \\
\hline 13 & Valerylcarnitine, Isovalerylcarnitine \\
\hline 14 & $\begin{array}{l}\text { Arachidyl carnitine, O-[(9Z)-17-carboxyheptadec- } \\
\text { 9-enoyl]carnitine }\end{array}$ \\
\hline 15 & Stearidonyl carnitine \\
\hline 16 & O-arachidonoylcarnitine \\
\hline 17 & (9Z)-3-hydroxyoctadecenoylcarnitine \\
\hline 18 & (9Z)-3-hydroxydodecenoylcarnitine \\
\hline 19 & Dodecanoylcarnitine, O-dodecanoylcarnitine \\
\hline 20 & 2-Hexenoylcarnitine \\
\hline 21 & (9Z,12Z,15Z)-3-hydroxyoctadecatrienoylcarnitine \\
\hline 22 & $\begin{array}{l}\text { Palmitoylcarnitine, (5Z)-13-carboxytridec-5- } \\
\text { enoylcarnitine }\end{array}$ \\
\hline 23 & (13Z,16Z)-docosadienoylcarnitine \\
\hline 24 & $\begin{array}{l}\text { Linoelaidyl carnitine, O-linoleoylcarnitine, 9,12- } \\
\text { Hexadecadienylcarnitine }\end{array}$ \\
\hline 25 & cis-5-Tetradecenoylcarnitine \\
\hline \multicolumn{2}{|c|}{ Phosphatidylcholines and Sphingomyelins } \\
\hline 26 & PC (32:0); PCo(33:0); $\mathrm{PCo}(32: 1) \mathrm{OH}$ \\
\hline 27 & PC (36:1); PCo(37:1); PCo(36:2)OH; PC (34:3)2OH \\
\hline 28 & $\mathrm{SM}(\mathrm{d} 18: 2 / 24: 1)$ \\
\hline 29 & PC (32:3); PCo(33:3) \\
\hline 30 & PC (42:5); PCo(42:6)OH; PC (40:7)2OH \\
\hline 31 & PC (44:8); PC (42:10)2OH \\
\hline 32 & SM (d18:2/18:1) \\
\hline 33 & PC (42:0); PCo(43:0); PCo(42:1)OH; PC (40:2)2OH \\
\hline 34 & PCo(44:4); PC (43:4) \\
\hline 35 & PC (50:0) \\
\hline 36 & PC (46:0) \\
\hline 37 & PCo(40:6); PC (39:6); PC (38:7)OH; PC (36:1)2OH \\
\hline 38 & PC (42:1); PCo(43:1); PCo(42:2)OH \\
\hline 39 & PC (44:7); PC (42:9)2OH \\
\hline
\end{tabular}

Table 1 The 25 most abundant lipid species in each class found in heatmaps (Figs. 2 and 3$)^{\text {a }}$ (Continued)

\begin{tabular}{ll}
\hline Number on Heatmap & Lipid Name \\
\hline 40 & PCo(42:1); PC (41:1); PC (40:2)OH \\
41 & PC (44:12); PC (43:5); PC (42:6)OH; PCp(42:6)2OH \\
42 & PCo(42:6); PC (41:6); PC (40:7)OH; PC (38:1)2OH \\
43 & PCo(34:3) \\
44 & PCo(32:2) \\
45 & PCo(40:0); PC (39:0) \\
46 & PC (52:0) \\
47 & PCo(40:1) \\
48 & PCo(38:5); PC (37:5); PC (34:0)2OH \\
49 & PCo(40:5); PC (39:5); PC (38:6)OH; PC (36:0)2OH; \\
50 & PCp(38:6)2OH \\
& PCo(38:0)
\end{tabular}

Phosphatidylinositols

\begin{tabular}{|c|c|}
\hline 51 & $\mathrm{PI}(36: 2)$ \\
\hline 52 & PI (12:0) \\
\hline 53 & $\mathrm{PI}(26: 0)$ \\
\hline 54 & PI (20:4) \\
\hline 55 & $\mathrm{PI}(36: 7)$ \\
\hline 56 & Plo (38:2), Plp (38:1); Plp (38:1); Plo (38:2) \\
\hline 57 & PI (40:1) \\
\hline 58 & $\mathrm{PI}(42: 7)$ \\
\hline 59 & Plp (42:2) \\
\hline 60 & $\mathrm{PI}(44: 1)$ \\
\hline 61 & PI (42:1) \\
\hline 62 & $\mathrm{PI}(44: 10)$ \\
\hline 63 & Plp (38:6) \\
\hline 64 & PI (44:5) \\
\hline 65 & Plo (36:1), Plp (36:0) \\
\hline 66 & PI (36:5) \\
\hline 67 & $\mathrm{PI}(30: 1)$ \\
\hline 68 & Plo (38:1), Plp (38:0) \\
\hline 69 & PI (42:8) \\
\hline 70 & PI (40:7) \\
\hline 71 & PI (20:5) \\
\hline 72 & PI (18:4) \\
\hline 73 & $\mathrm{PI}(14: 0)$ \\
\hline 74 & Plp (36:5) \\
\hline 75 & $\mathrm{PI}(42: 6)$ \\
\hline \multicolumn{2}{|c|}{ TAG 1} \\
\hline 7 & TAG(52:3)_FA 16:0 \\
\hline 7 & TAG(52:4)_FA 16:0 \\
\hline 7 & TAG(54:4)_FA 18:0 \\
\hline 7 & TAG(52:5)_FA 16:0 \\
\hline
\end{tabular}


Table 1 The 25 most abundant lipid species in each class found in heatmaps (Figs. 2 and 3) ${ }^{\mathrm{a}}$ (Continued)

\begin{tabular}{|c|c|}
\hline Number on Heatmap & Lipid Name \\
\hline 80 & TAG(54:5)_FA 18:1 \\
\hline 81 & TAG(54:6)_FA 18:1 \\
\hline 82 & TAG(52:2)_FA 18:0 \\
\hline 83 & TAG(54:3)_FA 16:0 \\
\hline 84 & TAG(50:0)_FA 16:0 \\
\hline 85 & TAG(50:3)_FA 16:0 \\
\hline 86 & TAG(50:2)_FA 16:0 \\
\hline 87 & TAG (49:7) \\
\hline 88 & TAG(48:1)_FA 18:0 \\
\hline 89 & TAG(54:8)_FA 16:1 \\
\hline 90 & TAG(56:8)_FA 16:1 \\
\hline 91 & TAG(56:2)_FA 18:0 \\
\hline 92 & $\operatorname{TAG}(53: 7)$ \\
\hline 93 & TAG(54:8)_FA 18:1 \\
\hline 94 & TAG(54:6)_FA 16:1 \\
\hline 95 & TAG(52:2)_FA 16:1 \\
\hline 96 & TAG(52:3)_FA 16:1 \\
\hline 97 & TAG(52:2)_FA 18:1 \\
\hline 98 & TAG(54:1)_FA 18:0 \\
\hline 99 & TAG(54:3)_FA 18:1 \\
\hline 100 & TAG(54:2)_FA 18:1 \\
\hline \multicolumn{2}{|l|}{ TAG 2} \\
\hline 101 & TAG(54:7)_FA 18:2 \\
\hline 102 & TAG(54:6)_FA 18:2 \\
\hline 103 & TAG(54:5)_FA 18:2 \\
\hline 104 & TAG(50:2)_FA 18:2 \\
\hline 105 & TAG(50:3)_FA 18:2 \\
\hline 106 & TAG(52:4)_FA 18:2 \\
\hline 107 & TAG(56:5)_FA 20:4 \\
\hline 108 & TAG(56:2)_FA 20:0 \\
\hline 109 & TAG(58:10)_FA 18:2 \\
\hline 110 & TAG(58:7)_FA 18:2 \\
\hline 111 & TAG(56:8)_FA 18:1 \\
\hline 112 & TAG(58:9)_FA 18:2 \\
\hline 113 & TAG(58:9)_FA 18:1 \\
\hline 114 & TAG(52:2)_FA 20:0 \\
\hline 115 & TAG(58:5)_FA 18:1 \\
\hline 116 & TAG(58:6)_FA 20:4 \\
\hline 117 & TAG(54:8)_FA 20:4 \\
\hline 118 & TAG(56:5)_FA 18:1 \\
\hline 119 & TAG(56:2)_FA 18:1 \\
\hline 120 & TAG(52:5)_FA 20:4 \\
\hline 121 & TAG(52:6)_FA 20:4 \\
\hline
\end{tabular}

Table 1 The 25 most abundant lipid species in each class found in heatmaps (Figs. 2 and 3) ${ }^{\mathrm{a}}$ (Continued)

\begin{tabular}{cl}
\hline Number on Heatmap & Lipid Name \\
\hline 122 & TAG(54:7)_FA 20:4 \\
123 & TAG(56:6)_FA 20:4 \\
124 & TAG(58:8)_FA 18:1 \\
125 & TAG(56:7)_FA 18:1 \\
\hline
\end{tabular}

Lipid names are listed as 2 or 3 letters for the class abbreviations followed by the number of carbon, a colon, and the number unsaturation in the fatty acyl chains (e.g. $P C(32: 3) . P C$ - phosphatidylcholine, $P C o$ - PC lipid with an alkyl ether substituent; PI - phosphatidylinositol, Plo: PI lipid with an alkyl ether substituent, PIp: PI lipid with a 1Z-alkenyl ether (plasmalogen) substituent, SM sphyngomyelin, TAG - triacylglycerol. The TAG lipids were profiled using a product ion related to one of the fatty acyl chains since this lipid class has no polar head as the phospholipids or another specific functional group. The targeted fatty acyl chain is indicated after the TAG abbreviation by_FA carbon number:unsaturation (e.g. $\operatorname{TAG}(52: 3) \_F A$ 16:0

occurs during aging [19]. In fact, the inability to recruit pre-adipocytes into the adipogenic lineage results in a failure of the tissue to expand and results in the hypertrophy of existing adipocytes [19]. It will, therefore, be informative to determine if the function of pre-adipocytes in peri-gonadal adipose tissue is compromised in mice of advanced reproductive age.

Adipocyte size is also related to the expression of cytokines, with hypertrophic cells favoring secretion of proinflammatory adipokines [16]. Interestingly, adipose tissue is a major site of accumulation of senescent cells with age. Cellular senescence occurs when cells enter a permanent cell cycle arrest due to stress, DNA damage, and/or potent mitogenic signals [37]. Because of the cumulative exposure to such stimuli with age, senescent cells accumulate in aging tissues and can actually fuel the aging process through acquisition of the Senescence Associated Secretory Phenotype (SASP) [21, 38, 39]. The SASP consists of secretion of cytokines, chemokines, growth factors, and proteases, which can cause chronic sterile inflammation and disrupt surrounding tissue structure and function [21, 38, 39]. Interestingly, TNF $\alpha$ and interleukin-6 (IL-6) are pro-inflammatory cytokines with high expression in senescent cells in rodent adipose tissue and also in ovarian tissue from mice of advanced reproductive age $[13,16,20]$. Senescence can spread across cells, so it will be interesting to determine what inflammatory factors the peri-gonadal adipose tissue is producing and secreting and whether there is a connection between the peri-gonadal adipose tissue and the ovary [20].

In addition to increased adipocyte size, we also noted distinct alterations in the lipid composition of peri-gonadal adipose tissue with advanced reproductive age with respect to acyl-carnitines, phosphatidylcholines and sphingomyelins, phosphatidylinositols, and TAGs. The most robust age-associated differences were observed in the TAGs. A major role of fat is to store calorically-rich fatty acids. 

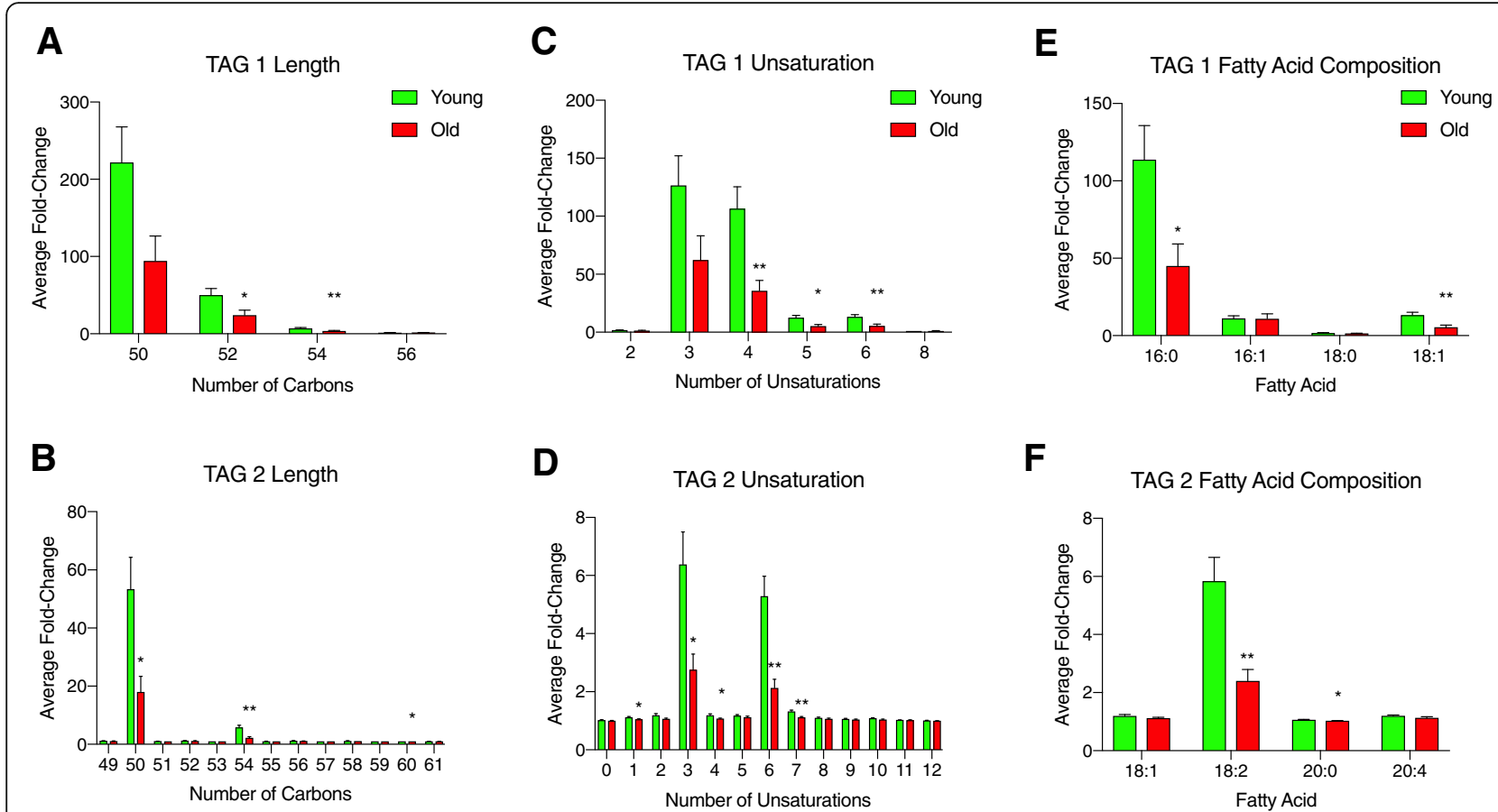

Fig. 4 Characteristics of the TAG species that change significantly with age in peri-gonadal adipose tissue. The average fold-change with regards to blank for TAG species by length (number of carbons) in peri-gonadal adipose tissue from reproductively young (green) and old (red) mice in the (a) TAG 1 method and (b) TAG 2 method. The average fold-change over blank for TAG species by number of unsaturation sites in perigonadal adipose tissue from reproductively young (green) and old (red) mice in the (c) TAG 1 method and (d) TAG 2 method (e). The average fold-change over blank for TAG species based on fatty acid composition in peri-gonadal adipose tissue from reproductively young (green) and old (red) mice in the (f) TAG 1 method and ( $\mathbf{g}$ ) TAG 2 method. Fatty acids are labeled as the length (number of carbons): number of unsaturation sites. Error bars represent standard error between samples. ${ }^{*} P$ value $\leq 0.05$ and ${ }^{*} P$ value $\leq 0.01$ by t-test

However, because fatty acids are reactive and cytotoxic molecules, cells store fatty acids as less reactive triglycerides. With age, however, there is a reduction in the incorporation of fatty acids as triglycerides [14]. This is consistent with our observation that there were quantitatively fewer TAGs per mg of peri-gonadal adipose tissue in reproductively old mice relative to young controls. The inability to store lipid can lead to the release of toxic free fatty acids that are then deposited into ectopic sites such as the liver, muscle, and pancreas, leading to lipotoxicity and organ damage $[16,19,20]$. Defining whether there is dysregulation of fatty acid storage and release in the peri-gonadal adipose tissue in reproductively old female mice warrants further investigation. This may in fact be the case, however, because we noted an increase in hepatic triglycerides in livers from mice of advanced reproductive age compared to controls (unpublished data). Additional quantitative MS

Table 2 The number of significantly different TAGs in peri-gonadal adipose tissue based on age

\begin{tabular}{lll}
\hline $\begin{array}{l}\text { Lipid } \\
\text { Subclass }\end{array}$ & $\begin{array}{l}\text { Young Peri-Gonadal Adipose } \\
\text { Tissue }\end{array}$ & $\begin{array}{l}\text { Old Peri-Gonadal Adipose } \\
\text { Tissue }\end{array}$ \\
\hline TAG 1 & 4 & 4 \\
TAG 2 & 3 & 149 \\
\hline
\end{tabular}

studies will also be required to determine what lipid species may be replacing the TAGs in the peri-gonadal adipose tissue with age that may account for the observed cell hypertrophy.

Age-associated changes in adipose tissue may have important ramifications as it relates to the ovary. For example, peri-ovarian tissue can regulate ovarian function in mice. Surgical removal of peri-ovarian tissue results in several phenotypes including delayed antral follicle growth, increased atretic follicle growth, and alterations in adipokines, growth factors, lipid accumulation, and expression of steroidogenic enzymes [40]. Thus, it is highly likely that age-associated changes in the peri-gonadal tissue could impact follicle dynamics and the ovarian environment. In addition, our findings may

Table 3 The amount of lipid per milligram of peri-gonadal adipose tissue in defined classes

\begin{tabular}{|c|c|c|}
\hline & $\begin{array}{l}\text { Young Peri-Gonadal } \\
\text { Adipose Tissue }\end{array}$ & $\begin{array}{l}\text { Old Peri-Gonadal } \\
\text { Adipose Tissue }\end{array}$ \\
\hline $\mu \mathrm{g}$ PC per mg/sample & 451.2 & 455.7 \\
\hline $\mu \mathrm{g}$ SM per mg/sample & 7.5 & 7.5 \\
\hline ug TAG per mg/sample & 33.1 & 10.2 \\
\hline
\end{tabular}



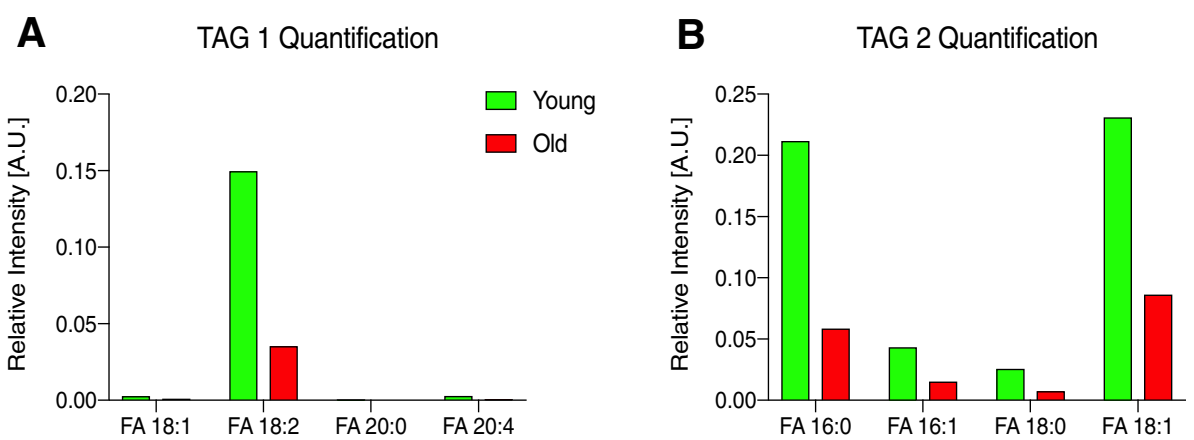

Fig. 5 Peri-gonadal adipose tissue from reproductively young mice has quantitatively more TAGs per milligram of tissue than reproductively old mice. The average fold-change with regards to blank for TAG species by fatty acid composition in peri-gonadal adipose tissue from reproductively young (green) and old (red) mice in the (a) TAG 1 method and (b) TAG 2 method. Fatty acids are labeled as the length (number of carbons): number of unsaturation sites

have clinical implications. Recent ovarian transplantation studies in mouse and xenotransplantation models have demonstrated that addition of adipose tissue-derived stem cells can improve the function of ovarian grafts as measured by the increase in the total volume of the ovary, progesterone and estradiol production, and number of follicles [41-46]. This improved function is thought to be due to factors that the adipose-derived mesenchymal stromal cells produce, including pro-angiogenic cytokines and anti-apoptotic and anti-inflammatory factors. These factors protect tissues against ischemia-reperfusion damage, which is the primary limitation of ovarian grafts [41-46]. Our findings are significant within this context because if co-transplantation of adipose tissue-derived stem cells and ovarian tissue is used clinically for ovarian tissue transplantation to restore endocrine function and/or fertility in women who have undergone gonadotoxic therapies, the age of the adipose tissue donor will be a critical variable to consider due to potential variations in adipose tissue architecture and composition.

There are several caveats surrounding our data which are important to consider. First, we do not know if the age-related changes in adipocyte architecture and lipid profiles we observed are specific to the peri-gonadal fat or to the adipose tissue in general. Second, humans do not have peri-gonadal fat per se, so whether analogous changes are conserved in omental adipose tissue will be important to investigate. The visceral omental fat most proximal to the ovary may influence reproductive function, and there is a precedent that dysfunctional adipose can negatively impact extra-adipose tissues and contribute to disease and possibly aging tissues. For example, inflammatory changes in adipose tissue and metabolic dysregulation observed in animal models of alcoholic liver disease (ALD) and obesity are associated with liver steatosis and inflammation [47-49]. Finally, more than $50 \%$ of dietary fat is stored in subcutaneous and visceral fat, with visceral fat being more efficient at storage [14]. Thus, some of the lipid changes we detected may also be influenced by diet. Our reproductively young and old mouse cohorts originated from different vendors and were initially fed different chows. However, the slight variations in fat content and fatty acid composition in the chows do not appear to correlate with the age-associated changes in peri-gonadal fat lipid profiles. Therefore, it appears that the diet effects are likely minor relative to the age effects. It is important to recognize, however, that diet can affect ovarian function. For example, low protein intake reduces mouse primordial follicle activation, and omega-3 polyunsaturated fatty acids supplements alter mouse follicular cell gene expression $[50,51]$. Thus, systematically determining the effect of specific diets on periovarian adipose tissue architecture and lipid profiles could be informative.

Although our primary interest is reproductive aging, we cannot overlook the interplay between aging and obesity. The reproductively old mice in our study weighed significantly more than our reproductively young mice as has been documented in other strains as well [12]. This weight increase was also accompanied by a larger mass of peri-gonadal fat with advanced reproductive age. These findings are consistent with what occurs in the human population with age where it is well-established that fat tissue mass and visceral adipose deposition increases during middle age $[17,20]$. There are several parallels between obesity and aging, including enhanced chronic inflammation, circulation of proinflammatory factors, ectopic fat deposition, and lipotoxicity [16, 20, 52]. Obesity also accelerates diseases common to old age. Interestingly, there are well-characterized effects of obesity on gamete quality and fertility in both preclinical and clinical settings $[53,54]$. For example, mouse models of obesity produce poor quality oocytes with aberrant maternal gene expression, dysfunctional mitochondria, and meiotic errors 
[55, 56]. In females undergoing Assisted Reproduction Technology cycles, oocyte and embryo counts per in vitro fertilization cycle are significantly lower in women with a body mass index above the normal range, especially in women with class-II/III obesity [57]. Given these parallels, it will be important to determine whether aging and obesity effects on reproductive function operate via independent or compounding mechanisms.

\section{Conclusions}

These findings provide clear evidence of age-associated physiological changes in peri-gonadal adipose tissue with regards to changes in adipocyte morphology and lipid profiles. Further investigation of the effect of peri-gonadal adipose tissue on the ovarian microenvironment may identify it as a promising target to improve reproductive function in the context of age-related infertility. For example, if aging fat tissue negatively impacts reproductive function, adipose transplantation or resection may be effective therapeutic strategies.

\section{Additional files}

Additional file 1: Table S1. Fat macronutrient content and fatty acid composition of mouse chows. Table S2. List of 160 TAGs that were significantly different with age ${ }^{1}$. (DOCX $30 \mathrm{~kb}$ )

Additional file 2: Figure S1. Ceramide, phosphatidylethanolamine, and phosphatidylserine lipid profiles are not different between peri-gonadal adipose tissue from reproductively young and old mice. 2D Principal component analysis (PCA) plot analysis of the lipid distribution between peri-gonadal adipose tissue from reproductively young (green) and old (red) mice for (A) ceramides, (B) phosphatidylethanolamines, and (C) phosphatidylserines. (DOCX $68 \mathrm{~kb}$ )

\section{Acknowledgements}

We thank Gillian R. Rubenstein for technical assistance.

\section{Funding}

This research was supported by the Eunice Kennedy Shriver National Institute of Child Health and Human Development (R01HD093726 to F.E.D and M.T.P.) and startup funds from the Department of Obstetrics and Gynecology (to F.E.D).

\section{Availability of data and materials}

The datasets used and/or analyzed during the current study are available from the corresponding author on reasonable request.

\section{Authors' contributions}

FED, CRF, and MTP designed the experiments. SSD, CRF, LTZ, and FED performed the experiments and analyzed the data. All authors contributed to manuscript writing and approved the final manuscript.

\section{Ethics approval and consent to participate}

Mice were used for this study, and all experiments were approved by the Institutional Animal Care and Use Committee (Northwestern University) and were performed in accordance with National Institutes of Health Guidelines.

\section{Consent for publication}

Not applicable

\section{Competing interests}

The authors declare that they have no competing interests.

\section{Publisher's Note}

Springer Nature remains neutral with regard to jurisdictional claims in published maps and institutional affiliations.

\section{Author details}

'Department of Obstetrics and Gynecology, Feinberg School of Medicine, Northwestern University, 303 E. Superior Street, Lurie 7-117, Chicago, IL 60611, USA. ${ }^{2}$ Center for Analytical Instrumentation Development, Department of Chemistry, Purdue University, West Lafayette, IN, USA. ${ }^{3}$ Bindley Bioscience Center, Purdue University, West Lafayette, IN, USA. ${ }^{4}$ Department of Pharmacology, Toxicology \& Therapeutics, University of Kansas Medical Center, Kansas City, KS, USA.

Received: 4 February 2019 Accepted: 7 May 2019

Published online: 12 June 2019

\section{References}

1. Lopez-Otin C, Blasco MA, Partridge L, Serrano M, Kroemer G. The hallmarks of aging. Cell. 2013;153(6):1194-217.

2. Broekmans FJ, Soules MR, Fauser BC. Ovarian aging: mechanisms and clinical consequences. Endocr Rev. 2009;30(5):465-93.

3. Heffner LJ. Advanced maternal age--how old is too old? N Engl I Med. 2004:351(19):1927-9.

4. Johnson JA, Tough S. Society of $\mathrm{O}$, Gynaecologists of $\mathrm{C}$. delayed childbearing. J Obstet Gynaecol Can. 2012;34(1):80-93.

5. Nagaoka SI, Hassold TJ, Hunt PA. Human aneuploidy: mechanisms and new insights into an age-old problem. Nat Rev Genet. 2012;13(7):493-504.

6. Duncan FE, Jasti S, Paulson A, Kelsh JM, Fegley B, Gerton JL. Age-associated dysregulation of protein metabolism in the mammalian oocyte. Aging Cell. 2017:16(6):1381-93.

7. Duncan FE, Hornick JE, Lampson MA, Schultz RM, Shea LD, Woodruff TK. Chromosome cohesion decreases in human eggs with advanced maternal age. Aging Cell. 2012;11(6):1121-4.

8. Chiang T, Duncan FE, Schindler K, Schultz RM, Lampson MA. Evidence that weakened centromere cohesion is a leading cause of age-related aneuploidy in oocytes. Current biology : CB. 2010;20(17):1522-8.

9. El-Hayek S, Yang Q, Abbassi L, FitzHarris G, Clarke HJ. Mammalian oocytes locally remodel follicular architecture to provide the Foundation for GermlineSoma Communication. Current biology : CB. 2018;28(7):1124-31 e3.

10. Nakagawa S, FitzHarris G. Intrinsically defective microtubule dynamics contribute to age-related chromosome segregation errors in mouse oocyte meiosis-I. Current biology : CB. 2017;27(7):1040-7.

11. May-Panloup P, Boucret L, Chao de la Barca JM, Desquiret-Dumas V, FerreL'Hotellier V, Moriniere C, et al. Ovarian ageing: the role of mitochondria in oocytes and follicles. Hum Reprod Update. 2016;22(6):725-43.

12. Hirshfeld-Cytron JE, Duncan FE, Xu M, Jozefik JK, Shea LD, Woodruff TK. Animal age, weight and estrus cycle stage impact the quality of in vitro grown follicles. Hum Reprod. 2011;26(9):2473-85.

13. Briley SM, Jasti S, McCracken JM, Hornick JE, Fegley B, Pritchard MT, et al. Reproductive age-associated fibrosis in the stroma of the mammalian ovary. Reproduction. 2016;152(3):245-60

14. Tchkonia T, Thomou T, Zhu Y, Karagiannides I, Pothoulakis C, Jensen MD, et al. Mechanisms and metabolic implications of regional differences among fat depots. Cell Metab. 2013;17(5):644-56.

15. Chusyd DE, Wang D, Huffman DM, Nagy TR. Relationships between rodent white adipose fat pads and human white adipose fat depots. Front Nutr. 2016;3:10

16. Zamboni M, Rossi AP, Fantin F, Zamboni G, Chirumbolo S, Zoico E, et al. Adipose tissue, diet and aging. Mech Ageing Dev. 2014;136(137):129-37.

17. Hunter GR, Gower BA, Kane BL. Age related shift in visceral fat. Int J Body Compos Res. 2010;8(3):103-8.

18. Schosserer M, Grillari J, Wolfrum C, Scheideler M. Age-induced changes in white, Brite, and Brown adipose depots: a mini-review. Gerontology. 2018; 64(3):229-36.

19. Palmer AK, Kirkland JL. Aging and adipose tissue: potential interventions for diabetes and regenerative medicine. Exp Gerontol. 2016;86:97-105.

20. Tchkonia T, Morbeck DE, Von Zglinicki T, Van Deursen J, Lustgarten J, Scrable $H$, et al. Fat tissue, aging, and cellular senescence. Aging Cell. 2010;9(5):667-84. 
21. Tchkonia T, Zhu Y, van Deursen J, Campisi J, Kirkland JL. Cellular senescence and the senescent secretory phenotype: therapeutic opportunities. J Clin Invest. 2013;123(3):966-72.

22. Hornick JE, Duncan FE, Sun M, Kawamura R, Marko JF, Woodruff TK. Ageassociated alterations in the micromechanical properties of chromosomes in the mammalian egg. J Assist Reprod Genet. 2015;32(5):765-9.

23. Duncan FE, Chiang T, Schultz RM, Lampson MA. Evidence that a defective spindle assembly checkpoint is not the primary cause of maternal ageassociated aneuploidy in mouse eggs. Biol Reprod. 2009;81(4):768-76.

24. Treff NR, Krisher RL, Tao X, Garnsey H, Bohrer C, Silva E, et al. Next generation sequencing-based comprehensive chromosome screening in mouse polar bodies, oocytes, and embryos. Biol Reprod. 2016;94(4):76.

25. Tao X, Landis JN, Krisher RL, Duncan FE, Silva E, Lonczak A, et al. Mitochondrial DNA content is associated with ploidy status, maternal age, and oocyte maturation methods in mouse blastocysts. J Assist Reprod Genet. 2017;34(12):1587-94

26. Dadarwal D, Adams GP, Hyttel P, Brogliatti GM, Caldwell S, Singh J. Organelle reorganization in bovine oocytes during dominant follicle growth and regression. Reprod Biol Endocrinol. 2015;13:124.

27. Dunning KR, Russell DL, Robker RL. Lipids and oocyte developmental competence: the role of fatty acids and beta-oxidation. Reproduction. 2014; 148(1):R15-27.

28. Cordeiro FB, Montani DA, Pilau EJ, Gozzo FC, Fraietta R, Turco EGL. Ovarian environment aging: follicular fluid lipidomic and related metabolic pathways. J Assist Reprod Genet. 2018;35(8):1385-93.

29. Rodriguez-Castelan J, Mendez-Tepepa M, Rodriguez-Antolin J, Castelan F, Cuevas-Romero E. Hypothyroidism affects lipid and glycogen content and peroxisome proliferator-activated receptor? Expression in the ovary of the rabbit. Reprod Fertil Dev. 2018.

30. Rodriguez-Castelan J, Mendez-Tepepa M, Carrillo-Portillo Y, AnayaHernandez A, Rodriguez-Antolin J, Zambrano E, et al. Hypothyroidism reduces the size of ovarian follicles and promotes hypertrophy of Periovarian fat with infiltration of macrophages in adult rabbits. Biomed Res Int. 2017;2017:3795950

31. Cordeiro FB, Ferreira CR, Sobreira TJP, Yannell KE, Jarmusch AK, Cedenho AP, et al. Multiple reaction monitoring (MRM)-profiling for biomarker discovery applied to human polycystic ovarian syndrome. Rapid Commun Mass Spectrom. 2017;31(17):1462-70

32. de Lima CB, Ferreira CR, Milazzotto MP, Sobreira TJP, Vireque AA, Cooks RG. Comprehensive lipid profiling of early stage oocytes and embryos by MRM profiling. J Mass Spectrom. 2018;53(12):1247-52.

33. Merriman JA, Lane SI, Holt JE, Jennings PC, Garcia-Higuera I, Moreno S, et al. Reduced chromosome cohesion measured by interkinetochore distance is associated with aneuploidy even in oocytes from young mice. Biol Reprod. 2013:88(2):31.

34. Bligh EG, Dyer WJ. A rapid method of total lipid extraction and purification. Can J Biochem Physiol. 1959;37(8):911-7.

35. Bonzon-Kulichenko E, Molto E, Pintado C, Fernandez A, Arribas C, Schwudke $D$, et al. Changes in visceral adipose tissue plasma membrane lipid composition in old rats are associated with adipocyte hypertrophy with aging. J Gerontol A Biol Sci Med Sci. 2018;73(9):1139-46.

36. Honek J, Seki T, Iwamoto H, Fischer C, Li J, Lim S, et al. Modulation of agerelated insulin sensitivity by VEGF-dependent vascular plasticity in adipose tissues. Proc Natl Acad Sci U S A. 2014;111(41):14906-11.

37. Campisi J. d'Adda di Fagagna F. cellular senescence: when bad things happen to good cells. Nat Rev Mol Cell Biol. 2007;8(9):729-40.

38. Freund A, Orjalo AV, Desprez PY, Campisi J. Inflammatory networks during cellular senescence: causes and consequences. Trends Mol Med. 2010;16(5): 238-46.

39. Zhu Y, Tchkonia T, Pirtskhalava T, Gower AC, Ding H, Giorgadze N, et al. The Achilles' heel of senescent cells: from transcriptome to senolytic drugs. Aging Cell. 2015

40. Yang $L$, Chen L, Lu X, Tan A, Chen Y, Li Y, et al. Peri-ovarian adipose tissue contributes to intraovarian control during folliculogenesis in mice. Reproduction. 2018;156(2):133-44

41. Shojafar E, Soleimani Mehranjani M, Shariatzadeh SMA. Adipose-derived mesenchymal stromal cell transplantation at the graft site improves the structure and function of autografted mice ovaries: a stereological and biochemical analysis. Cytotherapy. 2018;20(11):1324-36.

42. Shojafar E, Soleimani Mehranjani M, Shariatzadeh SMA. Adipose derived mesenchymal stem cells improve the structure and function of autografted mice ovaries through reducing oxidative stress and inflammation: a stereological and biochemical analysis. Tissue Cell. 2019;56:23-30.

43. Dolmans MM, Cacciottola L, Amorim CA, Manavella D. Translational research aiming to improve survival of ovarian tissue transplants using adipose tissue-derived stem cells. Acta Obstet Gynecol Scand. 2019;98(5):665-71.

44. Manavella DD, Cacciottola L, Payen VL, Amorim CA, Donnez J, Dolmans MM. Adipose tissue-derived stem cells boost vascularization in grafted ovarian tissue by growth factor secretion and differentiation into endothelial cell lineages. Mol Hum Reprod. 2019;25(4):184-93.

45. Manavella DD, Cacciottola L, Pomme S, Desmet CM, Jordan BF, Donnez J, et al. Two-step transplantation with adipose tissue-derived stem cells increases follicle survival by enhancing vascularization in xenografted frozen-thawed human ovarian tissue. Hum Reprod. 2018;33(6):1107-16.

46. Manavella DD, Cacciottola L, Desmet CM, Jordan BF, Donnez J, Amorim CA, et al. Adipose tissue-derived stem cells in a fibrin implant enhance neovascularization in a peritoneal grafting site: a potential way to improve ovarian tissue transplantation. Hum Reprod. 2018;33(2):270-9.

47. Maeda K, Cao H, Kono K, Gorgun CZ, Furuhashi M, Uysal KT, et al. Adipocyte/macrophage fatty acid binding proteins control integrated metabolic responses in obesity and diabetes. Cell Metab. 2005;1(2):107-19.

48. Wang ZG, Dou XB, Zhou ZX, Song ZY. Adipose tissue-liver axis in alcoholic liver disease. World J Gastrointest Pathophysiol. 2016;7(1):17-26.

49. Zhang $X$, Ji X, Wang Q, Li JZ. New insight into inter-organ crosstalk contributing to the pathogenesis of non-alcoholic fatty liver disease (NAFLD). Protein Cell. 2018;9(2):164-77.

50. Otte MV, Moreira F, Bianchi I, Oliveira J Jr, Mendes RE, Haas CS, et al. Effects of supplying omega-3 polyunsaturated fatty acids to gilts after weaning on metabolism and ovarian gene expression. J Anim Sci. 2019;97(1):374-84.

51. Zhuo $Y$, Hua L, Feng B, Jiang $X$, Li J, Jiang D, et al. Fibroblast growth factor 21 coordinates adiponectin to mediate the beneficial effects of low-protein diet on primordial follicle reserve. EBioMedicine. 2019;41:623-35.

52. Frasca D, Blomberg BB, Paganelli R. Aging, obesity, and inflammatory agerelated diseases. Front Immunol. 2017:8:1745.

53. Lane M, Zander-Fox DL, Robker RL, McPherson NO. Peri-conception parenta obesity, reproductive health, and transgenerational impacts. Trends Endocrinol Metab. 2015;26(2):84-90.

54. Broughton DE, Moley KH. Obesity and female infertility: potential mediators of obesity's impact. Fertil Steril. 2017;107(4):840-7.

55. Boudoures AL, Saben J, Drury A, Scheaffer S, Modi Z, Zhang W, et al. Obesity-exposed oocytes accumulate and transmit damaged mitochondria due to an inability to activate mitophagy. Dev Biol. 2017;426(1):126-38.

56. Reynolds KA, Boudoures AL, Chi MM, Wang Q, Moley KH. Adverse effects of obesity and/or high-fat diet on oocyte quality and metabolism are not reversible with resumption of regular diet in mice. Reprod Fertil Dev. 2015;27(4):716-24

57. Kudesia R, Wu H, Hunter Cohn K, Tan L, Lee JA, Copperman AB, et al. The effect of female body mass index on in vitro fertilization cycle outcomes: a multi-center analysis. J Assist Reprod Genet. 2018:35(11):2013-23.

Ready to submit your research? Choose BMC and benefit from:

- fast, convenient online submission

- thorough peer review by experienced researchers in your field

- rapid publication on acceptance

- support for research data, including large and complex data types

- gold Open Access which fosters wider collaboration and increased citations

- maximum visibility for your research: over $100 \mathrm{M}$ website views per year

At BMC, research is always in progress.

Learn more biomedcentral.com/submissions 